\title{
Modification of Electrically Conductive Adhesives for Better Mechanical and Electrical Properties
}

\author{
Pavel Mach ${ }^{1}$, Lukáš Richter ${ }^{1}$, Alena Pietriková ${ }^{2}$ \\ ${ }^{1}$ Department of Electrotechnology, Faculty of Electrical Engineering, \\ Czech Technical University in Prague, Prague, Czech Republic \\ mach@fel.cvut.cz,duraja@fel.cvut.cz \\ ${ }^{2}$ Department of Technologies in Electronics, Faculty of Electrical Engineering and Informatics, \\ Technical University of Kosice, Kosice, Slovakia \\ Alena.Pietikova@tuke.sk
}

\begin{abstract}
The goal of the work has been to check a possibility of improvement of electrical properties of electrically conductive adhesives with isotropic electrical conductivity to edge these properties toward to properties of solders. The electrical resistance and nonlinearity of I-V characteristic have been investigated. Two ways have been used for electrically conductive adhesive modification: addition of small amount of silver nanoparticles (grains) of three different dimensions into adhesive, and substitution of a part of filler flakes with the same amount of nanoparticles. It has been found that addition of silver nanoparticles increases the resistance and nonlinearity of adhesive. It has also been found that adhesives modified with nanoparticles have lower sensitivity to combined climatic ageing in comparison with adhesives without nanoparticles. The reason is other quality of contacts between flakes and contacts between nanoparticles.
\end{abstract}

\section{INTRODUCTION}

Electrically conductive adhesives can be dispensed in dots or lines of small width to make electrical connections for hybrid components, solder replacement, RFID assembly, or MEMs devices. These materials are, together with lead-free solders, eco-friendly substitutions of $\mathrm{Sn}-\mathrm{Pb}$ solders used for conductive joining in electronics in the past. With respect to the fact that $\mathrm{Pb}$ is a metal whose use is prohibited in RoHS Directive, it is necessary to find substitution of $\mathrm{Sn}-\mathrm{Pb}$ solders with comparable electrical as well as mechanical properties.

Two types of electrically conductive adhesives are used for conductive joining in electronics - adhesives with isotropic electrical conductivity (ICA) and with anisotropic electrical conductivity (ACA). Electrical conductivity of electrically conductive adhesives with isotropic electrical conductivity is lower in comparison with electrical conductivity of solders. Mechanical properties of adhesive joints are also worst than mechanical properties of soldered ones. Therefore these parameters of adhesives limit their use in some applications. The goal of the work has been to check if addition of small amount of silver nanograins can be used for improvement of electrical properties of ICA, especially its electrical resistance and nonlinearity of the current vs. voltage characteristic.

\section{Theoretical BACKGRound}

Comparison of electrical conductivity of selected materials is shown in Table 1.

Table 1. Electrical conductivity of selected materials

\begin{tabular}{|l|c|}
\hline \multicolumn{1}{|c|}{ Material } & $\begin{array}{c}\text { Rezistivity } \\
(\Omega \mathrm{cm})\end{array}$ \\
\hline Epoxy rezin & $10^{14}$ \\
\hline $\mathrm{ICA}-\mathrm{Au}$ filler & $10^{-3}$ \\
\hline $\mathrm{ICA}-\mathrm{Ag}$ filler & $10^{-4}$ \\
\hline $\mathrm{Sn}-\mathrm{Pb}$ solder - Sn60Pb40 & $3,0.10^{-5}$ \\
\hline $\mathrm{Au}$ & $2,4.10^{-6}$ \\
\hline $\mathrm{Ag}$ & $1,6.10^{-6}$ \\
\hline
\end{tabular}


ICAs consist of two components - binder and filler. Binder creates an insulating matrix. Epoxy resin, silicon resin or polyamide resin is used as the binder usually. Filler particles create a conductive net. Ag flakes, Au flakes or AgPd flakes are used as the filler the most often. $\mathrm{Ag}$ is a material with high electrical and thermal conductivity; however, mobility of its ions is very high. This property can cause problems if an adhesive joint is exposed at the environment with high humidity and if the DC voltage is applied. The $\mathrm{Ag}$ ions can migrate out of the adhesive joint and cause conductive bridges between near joints. Therefore $\mathrm{Au}$ or $\mathrm{AgPd}$ are sometimes used instead Ag, despite the fact that their price is higher.

Three mechanisms influence the total electrical resistance of the adhesive in general:

- Conductivity of filler material.

- Tunneling effect between filler particles.

- Restriction resistance between filler particles.

The restriction resistance depends on the shape of filler particles. If balls are used, the restriction resistance is a significant part of the total resistance of adhesive. If flakes are used, contacts between flakes have bigger area and are more frequent, and therefore the contribution of the restriction resistance to the total electrical resistance of adhesive is low. Therefore contribution of the constriction resistance can be neglected for ICAs [1].

Electrical conductivity of ICAs depends on many parameters. Concentration of filler particles in adhesive has dominant influence on electrical conductivity. Usually this concentration is between 60 to $80 \%$ (wt.).

If filler particles are spherical and if it is assumed that the contact area between the neighboring particles is a circle with a radius $a$, then the contact resistance can be expressed as:

$$
R=R_{c}+R_{\varepsilon}=\frac{e}{2 a}
$$

Where:

$$
\begin{aligned}
& R_{c} \quad \ldots \quad \text { constriction resistance, } \\
& R_{t} \quad \ldots \quad \text { tunnel resistance, } \\
& \text { a } \quad \ldots \quad \text { radius of a contact circle, } \\
& \rho \quad \ldots \quad \text { resistivity of balk material of } \\
& \text { particles, } \\
& \sigma \quad \ldots \quad \text { tunnel resistivity (in } \Omega \mathrm{m}^{2} \text { - the resistance per } \\
& \mathrm{m} 2 \text { of the insulating barrier). }
\end{aligned}
$$

For calculation of the bulk resistance of ICA different types of model are used. A Boolean model is used very often. It is assumed that the filler particles are completely blended in the resin. The principle of modeling is to generate a set of uniform randomly distributed coordinate values for all centroids of the particles. This model is not good because overlaps of particles are allowed and many of the spheres overlap. Therefore improved models are usually used where overlapping of spheres is impossible [2]. Then a microstructure model has to be simulated to find 3D distribution of filler particles in the resin.

If flakes are uses as filler particles, the solution is substantially more complicated. Contrary to the previous case the shape and position of flakes are described by 7 parameters, i.e. $x, y$ and $z$ coordinate of the flake center, the flake width $2 w_{0}$, the flake length $2 l_{0}$, the flake thickness $2 t_{0}$ and the flake alignment angle $\alpha$. It is assumed that the contacts between flakes are rectangular. With respect to the fact that the flakes can touch in more than 1 contact area it is assumed that the tunnel resistance can be neglected. The formula for calculation of the contact resistance between two flakes is:

$$
R=R_{t}=\begin{aligned}
& \rho \\
& A
\end{aligned}
$$

Where:

$\begin{array}{lll}A & \ldots & \text { contact area of all contacts between }\end{array}$ two flakes.

The resistivity of the adhesive can now be simulated using a finite elements method. ANSYS can be used for such the simulation.

Different types of nanoparticles are used to improve electrical, mechanical, or thermal properties of ICAs. Electrically conductive balls, metal nanowires, carbor nanotubes or carbon nanotubes filled with $\mathrm{Ag}$ are used for improvement of electrical conductivity, $\mathrm{SiO}_{2}$ particles for improvement of thermal conductivity.

If filler particles are of two types, e.g. flakes with a small part of nanoparticles, the percolation threshold such an adhesive decreases. It means that the process of aggregation proceed by other way in comparison with the aggregation process in adhesives filled with the particles of one type only. It is assumed that the nanoparticles create new physico-chemical bonds with epoxy resin. These bonds can increase the contact area and then minish the interspace of $\mathrm{Ag}$ particles [3]. 
This way the resistivity of adhesive should decrease. On the other hand the growing number of interconnections caused with added nanoparticles will increase electrical resistance of adhesive. Increasing of the electrical resistance after addition of nanoparticles into ICA is mostly observed.

\section{EXPERIMENTAL}

One-component isotropic electrically conductive adhesive formed of bisphenol-epoxy resin and silver flakes (dimensions 6 to $8 \mu \mathrm{m}$ ) has been used as the basic adhesive for experiments based on filling of adhesive with silver nanoparticles [4]. Concentration of silver flakes in this basic adhesive has been $75 \%$ (wt.)

Nanoparticles have been grains of 3 dimensions: 3 to $55 \mathrm{~nm}, 6$ to $8 \mathrm{~nm}$ and 80 to $100 \mathrm{~nm}$. Filling has been carried out by two ways:

- A part of silver flakes has been substituted with nanoparticles to preserve the constant contents of silver in adhesive.

- The total amount of silver in adhesive has been increased by addition of nanoparticles,

Samples of modified basic adhesive are presented in Table 2.

Table 2. Adhesives under test, basic adhesive: ICA,bisphenoí-epoxy resin, silver flakes 6 to $8 \mu \mathrm{m}$, $75 \%$ wt.

\begin{tabular}{|c|c|c|c|}
\hline$\underset{\#}{\text { Sample }}$ & $\begin{array}{l}\text { Nanoparticles } \\
\text { dimensions } \\
(\mathrm{nm})\end{array}$ & $\begin{array}{l}\text { Concent- } \\
\text { ration } \\
\% \text { (wt.) }\end{array}$ & Processing \\
\hline s1 & $(3-55)$ & 10 & \multirow{3}{*}{ 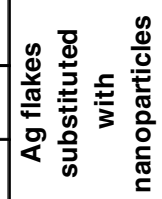 } \\
\hline S2 & $(3-55)$ & 20 & \\
\hline S3 & $(3-55)$ & 30 & \\
\hline S4 & $(80-100)$ & 3,8 & \multirow{4}{*}{ 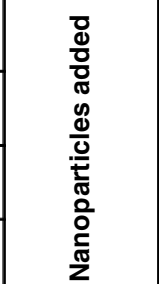 } \\
\hline S5 & $(6-8)$ & 3,8 & \\
\hline S6 & $(80-100)$ & 7,4 & \\
\hline S7 & $(6-8)$ & 7,4 & \\
\hline
\end{tabular}

\section{Measurements ANd TeSting}

Sets of 42 adhesive joints have been formed of every sample of modified adhesive and of the basic adhesive, too. Measured values have been processed as follows: five lowest and five highest values have been neglected, average values have been calculated of odd 32 measured values.

Electrical resistance and nonlinearity of the current vs. voltage characteristic of adhesive joints, formed of these adhesives, have been investigated.

The electrical resistance has been measured using an MCP TH 2818 Automatic Component Analyzer. Nonlinearity of the current vs. voltage characteristic has been measured using equipment designed and realized at the Department of Electrotechnology. The tensile strength has been measured using a tensile strength tester Vectra 2000.

Following climatic load has been applied on adhesive joints formed of the adhesive samples S1 to S3:

- Temperature $125 \mathrm{oC}$ for 700 hours.

- Humidity $99 \%$ RH for 700 hours.

- Combined climatic load $80{ }^{\circ} \mathrm{C} / 80 \%$ RH for 700 hours.

\section{ReSUlts AND Discussion}

Resistances of the joints formed of different samples of basic adhesive modified according to the Table 2 are shown in Figure 1.

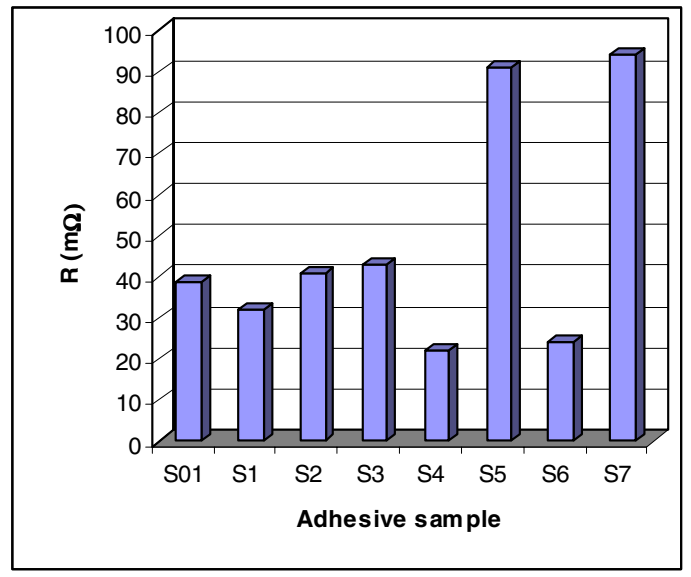

Figure 1. Resistance of modified adhesives, S01 is the resistance of basic adhesive used for modification 
Nonlinearity of the joints formed of different samples of basic adhesive modified according to the Table 2 is shown in Figure 2.

Resistances and nonlinearity changes of the joints formed of samples of basic adhesive modified according to the Table 2 after different types of climatic load are shown in Figure 3 to Figure 8.

It has been found that addition of silver nanoparticles into the adhesive does not cause increase of adhesive conductivity. The reason is higher number of contacts in conductive net created by particles of filler after addition of nanoparticles. It has also been found that distribution of nanoparticles in adhesive is not homogeneous.

It has also been found that some nanoparticles create clusters, which do not contribute to improvement of electrical conductivity of adhesive.

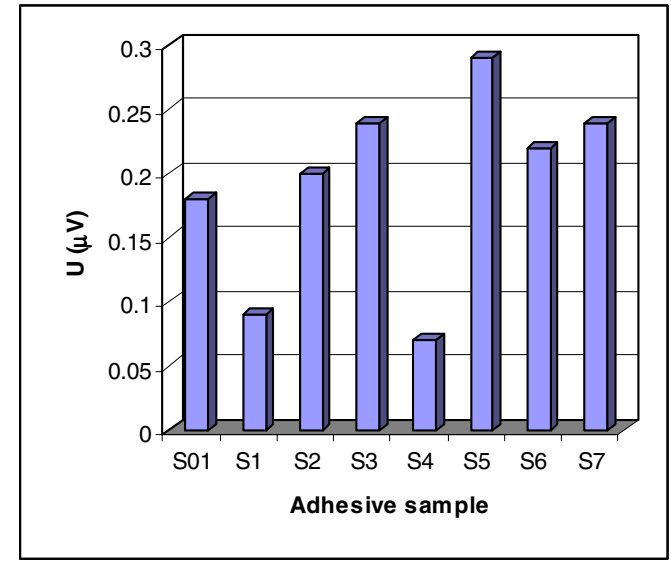

Figure 2. Nonlinearity of modified adhesives, S01 is nonlinearity of basic adhesive used for modification

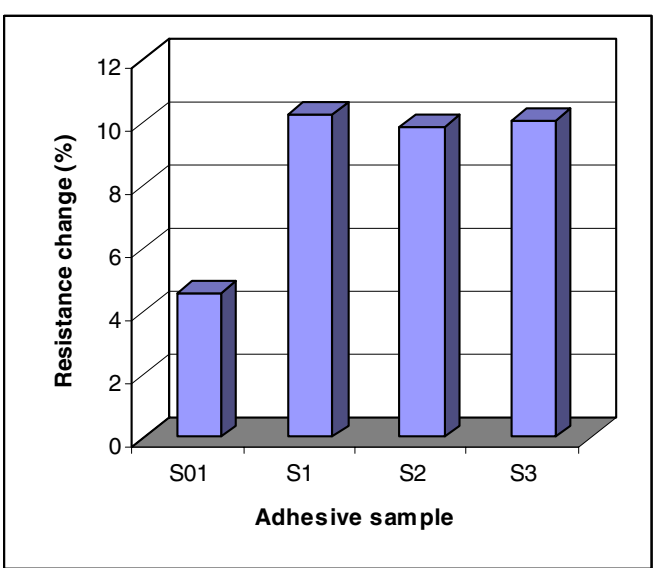

Figure 3 Resistance change of modified adhesives after thermal aging $\left(125^{\circ} \mathrm{C}, 700\right.$ hours $), \mathrm{S} 01$ is the resistance change of basic adhesive used for modification

The resistance of modified adhesives depends strongly on dimensions of nanoparticles added into the adhesive. Figure 1 shows that such the modification, when exact amount of Ag flakes are substituted with nanoparticles of the same weight, does not change the resistance of adhesive in wide range of such the substitution, up to $30 \% \mathrm{wt}$.

The reason is that no additional bridges among silver flakes have been created by these nanoparticles.

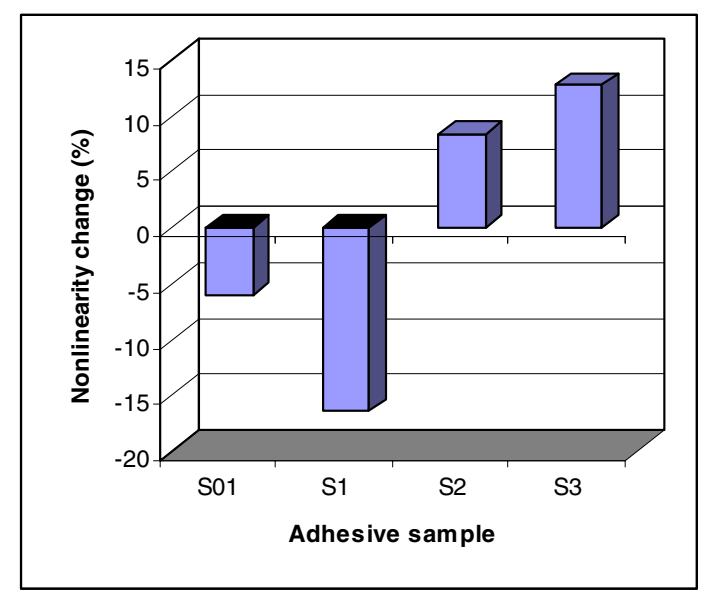

Figure 4. Nonlinearity change of modified adhesives after thermal aging $\left(125^{\circ} \mathrm{C}, 700\right.$ hours $), \mathrm{S} 01$ is nonlinearity change of basic adhesive used for modification

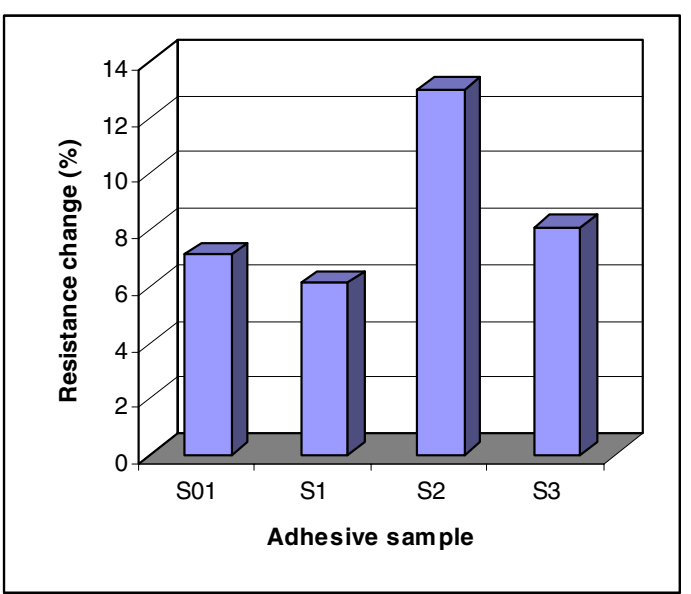

Figure 5. Resistance change of modified adhesives after humidity aging (100\% RH, 700 hours), S01 is the resistance change of basic adhesive used for modification 
On the other hand we have found that addition of small amount of nanoparticles into the adhesive and increase of its $\mathrm{Ag}$ contents can change its resistance significantly (see samples S4 to S7 in Figure 1).

The change of the resistance of adhesive joints depends on dimensions of the nanoparticles. When the nanoparticles of higher dimensions have been added, the joint resistance has decreased, when nanoparticles of small dimensions have been added, the resistance has increased. Explanation of this dependence is as follows: bigger nanoparticles can create parallel bridges between flakes with higher probability than smaller ones, because probability of their clustering is lower in comparison with the smaller nanoparticles.

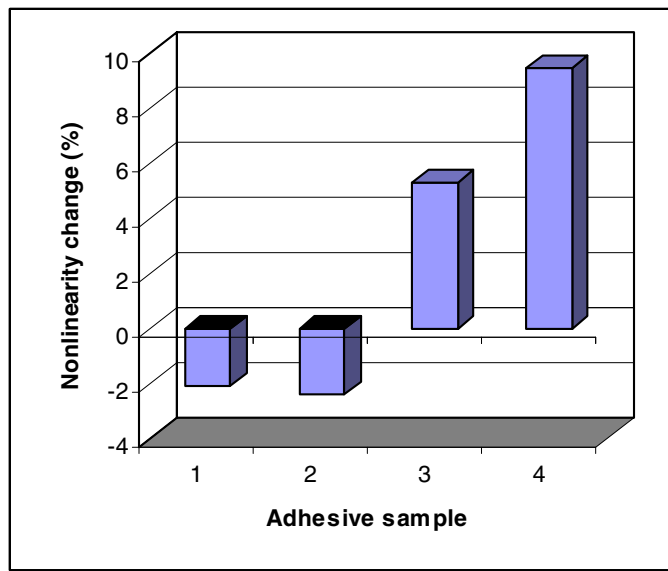

Figure 6. Nonlinearity change of modified adhesives after humidity aging (100\% RH, 700 hours), S01 is nonlinearity change of basic adhesive used for modification

Probability of clustering of smaller nanoparticles is higher and they create more contacts inside the conductive net. That is the reason why the resistance of adhesive joints increases.

Nonlinearity of the joints has also been influenced with addition of the nanoparticles. It has been assumed that the trend of nonlinearity will copy the trend of the resistance.

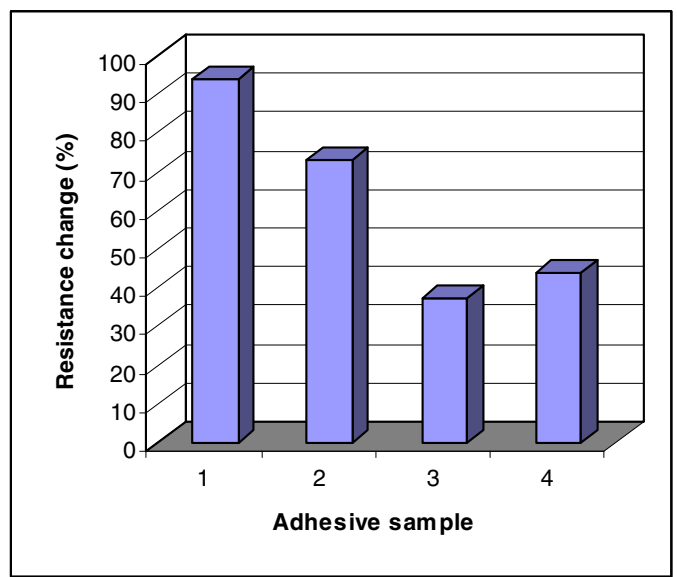

Figure 7. Resistance change of modified adhesives after thermal/humidity aging $\left(80{ }^{\circ} \mathrm{C} / 80 \% \mathrm{RH}, 700\right.$ hours), S01 is the resistance change of basic adhesive used for modification

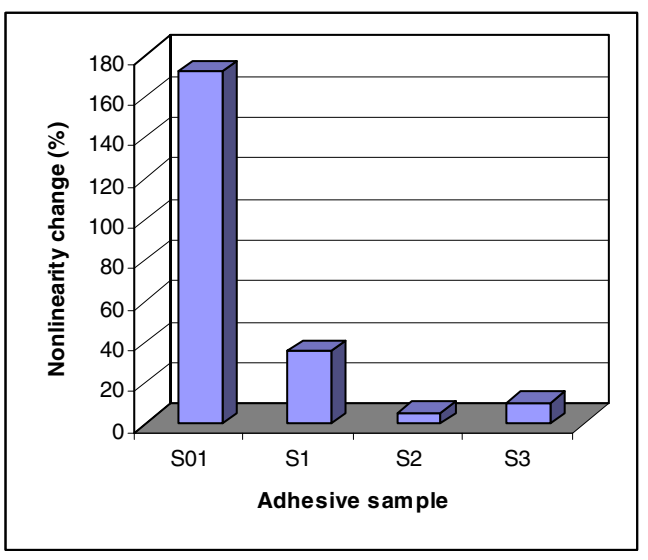

Figure 8. Nonlinearity change of modified adhesives after thermal/humidity aging $\left(80{ }^{\circ} \mathrm{C} / 80 \% \mathrm{RH}, 700\right.$ hours), $\mathrm{S} 01$ is the resistance change of basic adhesive used for modification

This assumption has been confirmed for the sample S4, but it has not been confirmed for the sample S6. The reason can be that new contacts created in adhesive by nanoparticles have higher nonlinearity in comparison with the contacts between flakes.

The resistance of adhesive after thermal aging has increased due to partial damage of contact between the conductive particles caused by oxidation or other chemical process. Addition of nanoparticles has 
reduced resistivity against thermal ageing. It has been found (see Figure 4) that the higher is the contents of nanoparticles, the higher is increase of nonlinearity after thermal ageing. The reason is the increase of total number of contacts in the conductive net.

Humidity aging has shown low influence of nanoparticles on the change of the resistance (see Figure 5), the changes of nonlinearity are similar to the changes of nonlinearity found after thermal ageing.

Positive influence of nanoparticles, added into the electrically conductive adhesive, on the resistance and nonlinearity of adhesive, has been found for combined climatic ageing. It has been detected lower increase of the resistance and significantly lower increase of nonlinearity after combined thermal/humidity ageing for the samples with nanoparticles in comparison with the samples without them. The reason is that the changes caused with this type of ageing are very high in comparison with the changes caused with other types of ageing. The changes of contact nonlinearity among nanoparticles are lower with respect of lower contact area between nanoparticles in comparison with the contact area between flakes

\section{Conclusions}

Electrical properties, the resistance and nonlinearity of adhesive joints formed of ICA modified with silver nanoparticles have been examined. It has been found that the nanoparticles do not improve electrical properties of adhesive joints, but improve their resitance against combined climatic ageing. The reason is that the contact areas between nanoparticles are substantially smaller in comparison with the contact areas between flakes. Therefore the contact pressure between nanoparticles is higher than between flakes and the contact is more resistive to external climatic load.

\section{ACKNOWLEDGMENTS}

The work has been carried out as a part of a project "Diagnostics of Materials", number MSM6840770021

\section{REFERENCES}

[1] Su, B.: Electrical, Thermomechanical and Reliability Modeling of Electrically Conductive Adhesives. Disertation. Georgia Institute of Technology. 2006

[2] Mündlein, M., Nicolics, J.: Modeling of Particle Arrangement in a Isotropically Conductive Adhesive Joint, IEEE Transaction on Components and Packaging Technologies, vol.28, no.4, December 2005, pp. 765-770.

[3] Quinghua Li, Jianhua, Zhang: Effects fo Nano Fillers on the Conductivity and Reliability of Isotropic Conductive Adhesives (ICAs). Key Engineering Materials Vols., 353-358, 2007, pp 2789-2882

[4] Radev, R.: Study of Properties of Some Types of Electrically Conductive Adhesives, Diploma Thesis, CTU Prague, 2007 